\title{
Opportunistic screening for colorectal neoplasia in Singapore using faecal immunochemical occult blood test
}

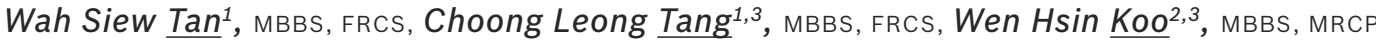

\begin{abstract}
INTRODUCTION The use of faecal immunochemical occult blood test (FIT) has been reported to decrease mortality from colorectal cancer. The Singapore Cancer Society (SCS) gives out FIT kits to encourage opportunistic screening of colorectal cancer. Any Singapore citizen or permanent resident aged $\geq 50$ years is eligible to receive two FIT kits. Participants with at least one positive FIT are referred for further evaluation. We aimed to analyse the results of SCS data from the year 2008.

METHODS The factors evaluated included compliance, positive test rate (PR) and positive predictive value (PPV) of FIT. RESULTS 20,989 participants received 41,978 kits in 2008 . Compliance was $38.9 \%$, with 8,156 participants returning at least one kit. $8 \%$ of participants tested positive, and $75 \%$ of these test-positive participants agreed to undergo further investigations. 33 participants had colorectal cancers, 45 had advanced polyps $(\geq 1 \mathrm{~cm})$ and 90 had polyps < $1 \mathrm{~cm}$. Histologically, 114 polyps were adenomatous, 20 were hyperplastic and 1 was serrated. PPV of colorectal neoplasia for those who underwent further colonoscopy was $34 \%$. Over half of the participants who had only one positive test had colorectal neoplasia.

CONCLUSION PR and PPV of FIT in our study were comparable to that in the literature. However, compliance was low and a quarter of all participants who tested positive refused further investigations. Extensive population education programmes are required to improve compliance and tackle inhibitions among the masses. It is also important to take steps to enhance the cost effectiveness of future screening programmes.
\end{abstract}

Keywords: colorectal cancer, faecal occult blood test, screening

\section{INTRODUCTION}

The incidence of colorectal cancer in Singapore has been increasing over the last ten years. It is the most common cancer in Singapore when the combined incidences for both genders are taken into account. ${ }^{(1)}$ Population-based randomised trials have shown that the use of faecal occult blood test (FOBT) reduces mortality from colorectal cancer, ${ }^{(2-6)}$ with a possible reduction in cancer incidence being effected through the early detection and removal of colorectal adenomas. ${ }^{(7)}$ Earlier detection of colorectal cancers may result in less invasive surgery and decreased stoma formation. ${ }^{(7,8)}$ In countries such as the United Kingdom and Italy, FOBT is used in population-based national colorectal cancer screening programmes. In Singapore, a national programme for the screening of colorectal cancer was only established in July 2011, when a population screening programme was initiated by the Health Promotion Board, Singapore. However, various organisations in Singapore, the largest being the Singapore Cancer Society (SCS), have been encouraging the public to undergo voluntary screening for colorectal cancer even prior to this time by issuing free FOBT kits. Such initiatives were in addition to efforts aimed at educating the public on risk factors, symptomatology, screening, diagnosis and treatment of colorectal cancer.
Since 2003, the SCS has been giving out free faecal immunochemical occult blood test (FIT) kits (OC-Light, Nagase, Singapore) to Singapore citizens and permanent residents aged 50 years and above. To date, the SCS is the largest distributor of FOBT kits in Singapore. Funding for these kits is obtained through a grant donation to the SCS. The authors contend that analyses of the results of the society's colorectal cancer screening programme would allow us to evaluate the feasibility of a nationwide population-based screening programme in Singapore, as well as the potential obstacles that may be faced during its implementation. In addition, the results of such a study could be considered a pilot study evaluating the expected results in the local setting. The year in which the highest number of FOBT kits was given out by the SCS was 2008 (based on the years for which data were available; data from 2009 were not yet ready). Therefore, the authors aimed to analyse the results of the SCS data obtained from the opportunistic screening conducted in 2008.

\section{METHODS}

FIT kits were distributed free of charge at the SCS office and through participating stores such as the Guardian pharmacy outlets.

${ }^{1}$ Department of Colorectal Surgery, Singapore General Hospital, ${ }^{2}$ Department of Medical Oncology, National Cancer Centre Singapore, ${ }^{3}$ Singapore Cancer Society, Colorectal Cancer Screening Committee

Correspondence: A/Prof Choong Leong Tang, Senior Consultant, Department of Colorectal Surgery, Singapore General Hospital, Outram Road, Singapore 169608. tang.choong.leong@sgh.com.sg 
The assay of the FIT kit is based on latex particle-linked antigenantibody reaction, which uses murine antibodies to specifically detect human haemoglobin $(\mathrm{Hb})$ in stool. Two kits were given free to each individual. Although individuals with a family history of colorectal cancer were discouraged from participating in the programme, several people managed to obtain the kits.

Each participant was taught how to collect the stool samples in the correct manner and to return the kits within a time frame of up to a week - through designated drop boxes at certain locations in Singapore, by post to the SCS office in sealed waterproof envelopes or returned directly to the society's office premises. The samples were processed in certified pathology laboratories in Singapore. Test results were available $4-6$ weeks after the return of kits. The level of occult blood for a positive test was assigned as $100 \mathrm{ng} \mathrm{Hb} / \mathrm{mL}$.

In the event of a negative result, participants were notified by post and advised to repeat the test in one year's time. In the event of a positive result, participants were notified both by post and telephone, and advised to seek further treatment. Referral letters were prepared and appointments with specialists were set up for these participants. Participants who were reluctant to seek further treatment were counselled, and attempts were made to persuade them to change their minds. All participants were encouraged to obtain copies of their investigation results, and additional phone calls were made to each participant to follow up on the results of further investigation.

The results of this voluntary opt-in screening programme from January 2008 to December 2008 were analysed. The factors evaluated included compliance rate (return rate) of the kits, the percentage of positive tests (test positivity rate [PR]) and positive predictive value (PPV) of the FIT. Data on participants who underwent further investigations following positive tests were further analysed.

\section{RESULTS}

A total of 41,978 kits were distributed to 20,989 individuals over a one-year period in 2008. 15,497 kits from 8,156 people were returned and analysed, giving a compliance rate of $38.9 \%$. Positive FIT results were obtained in 808 kits from 663 participants - 594 participants returned both kits, while 69 returned only one of the two kits issued to them. Among participants who returned both kits, 449 had positive results in only one kit and 145 had positive results in both kits. The PR among screened participants who returned the kits was $8.1 \%$.

Out of 663 participants with positive test results, 494 $(74.5 \%)$ agreed to undergo further investigations - colonoscopy ( $n=474)$, or barium enema or computed tomographic colonography $(n=20)$. However, 169 participants refused further investigations despite multiple attempts to persuade them to do otherwise. The data of the 494 participants who underwent further investigations were analysed. 273 (55.3\%) were women and $221(44.7 \%)$ were men, with a median age of 59 (range 34-89) years. Most of the participants were of Chinese
Table I. Pathology results of participants with positive FIT ( $n=494)$.

\begin{tabular}{lc}
\hline Pathology & No. (\%) \\
\hline Normal & $221(44.7)$ \\
Polyp & $135(27.3)$ \\
Colorectal cancer & $33(6.7)$ \\
Diverticular disease & $30(6.1)$ \\
Piles or haemorrhoids & $67(13.6)$ \\
Ulcer/infective colitis & $8(1.6)$ \\
\hline
\end{tabular}

Table II. Surgeries performed and the stage of cancers $(n=33)$.

\begin{tabular}{lc}
\hline Variable & No. (\%) \\
\hline AJCC staging & \\
Stage I & $11(33.3)$ \\
Stage II & $5(15.2)$ \\
Stage III & $17(51.5)$ \\
Stage IV & 0 \\
Type of surgery & \\
Right hemicolectomy & $6(18.2)$ \\
Left hemicolectomy & $3(9.1)$ \\
High anterior resection & $15(45.5)$ \\
Low anterior resection & $7(21.1)$ \\
Abdominoperineal resection & $1(3.0)$ \\
Total colectomy & $1(3.0)$ \\
\hline
\end{tabular}

AJCC: American Joint Committee on Cancer

Table III. Details of polyps $(n=135)$.

\begin{tabular}{lc}
\hline Variable & No. (\%) \\
\hline Histological findings & \\
Adenoma* & $114(84.4)$ \\
Hyperplastic & $20(14.8)$ \\
Serrated adenoma & $1(0.7)$ \\
No. of polyps & \\
$1-2$ & $116(85.9)$ \\
$>2$ & $19(14.1)$ \\
\hline
\end{tabular}

*Tubular, tubulovillous and villous

ethnicity (96.8\%). The findings of these further investigations are summarised in Table I.

Invasive colorectal cancer was diagnosed in 33 (6.7\%) FITpositive participants. The majority (26/33) of these participants had left-sided cancers, while only seven participants had right-sided cancers. All the participants underwent resection. About half of the participants $(n=17)$ diagnosed with cancer had American Joint Committee on Cancer (AJCC) stage III disease. None of the patients had metastatic disease at diagnosis. The details of surgeries performed and the patients' disease stage are summarised in Table II. One or more polyps were found on colonoscopy in 135 (27.3\%) participants. Nearly $85 \%$ of these polyps were adenomatous, and nearly $86 \%$ of the 135 participants had 1-2 polyps synchronously (Table III). Among the remaining 326 participants, 221 (44.7\%) had no pathology found on evaluation, 30 (6.1\%) had diverticular disease, 67 (13.6\%) had haemorrhoids and 8 (1.6\%) had inflammatory/infective colitis. Fig. 1 illustrates the sequence of screening adopted in this study. 


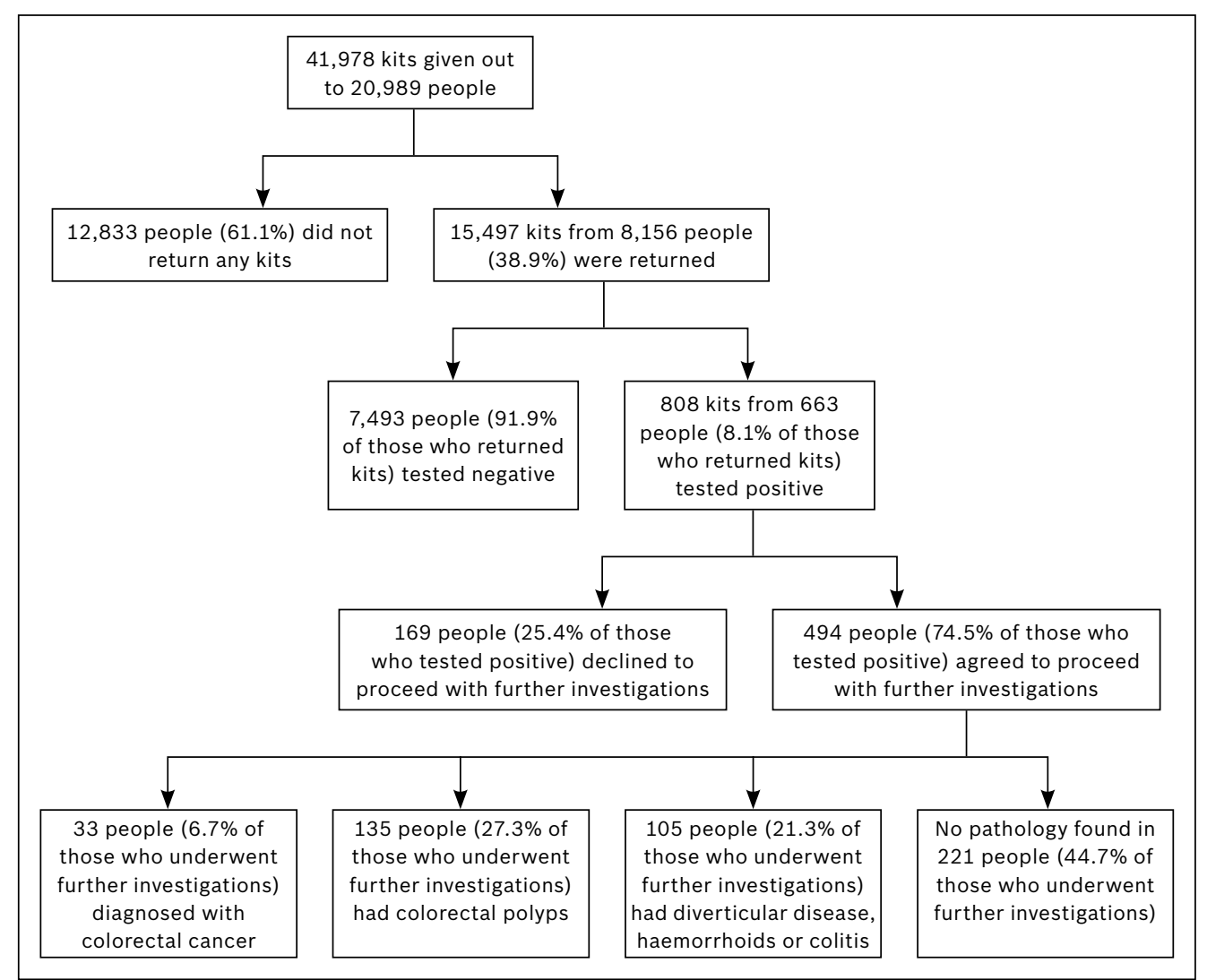

Fig. 1 Flowchart shows the screening sequence adopted in the study.

Overall, the PPV of FIT for colorectal neoplasia in our study was $34 \%$. More than half of the participants who had only one positive test were diagnosed with colorectal neoplasia - $55.5 \%$ of participants had at least one adenoma, while $1.9 \%$ had colorectal carcinoma (data not shown).

\section{DISCUSSION}

Colorectal cancer is the most common cancer in Singapore, but unlike breast or cervical cancer, which has established screening programmes, population screening for colorectal cancer in Singapore only began in July 2011. Multiple large studies performed in other countries have shown that colorectal cancer screening using FOBT decreases colorectal cancer mortality. ${ }^{(2-6)}$ The reported specificity and sensitivity of FIT for colorectal cancer is $93.4 \%-96.9 \%$ and $61.5 \%-87.5 \%$, respectively. ${ }^{(9-13)}$ While two previous studies have also evaluated the use of FOBT in Singapore, their sample sizes were smaller than that in our study. ${ }^{(11,14)}$

We found the compliance rate to be relatively low in our series. The possible reasons for such poor compliance could include feeling queasy about collecting stool samples or a lack of effort on the part of the individual to return the test kits. FOBT differs from a mammogram or Pap smear, in that the test is performed by the screened individual and the samples have to be returned by the individual. In addition, our results seem to indicate that non-Chinese do not voluntarily come forward for screening, as seen in the large proportion of Chinese participants (96.8\%) in our study. Thus, it may be necessary to take steps to address the ethnic inhibitions among other races, so as to increase their compliance rates.

In our study, nearly a quarter of the participants who tested positive on FIT refused to undergo further investigations. Ad-hoc interviews with those who declined further tests revealed common refrains such as 'too busy to undergo colonoscopy', 'too costly', 'if I am well, why go for colonoscopy?' and 'cannot be bothered'. Previous studies have indicated that the general public may be more inclined to accept screening if they have current information about other patients' experiences of colonoscopy and are more knowledgeable regarding the detection and treatment of bowel cancers. ${ }^{(15,16)}$ This may signal the need for more extensive mass population education programmes aimed at improving compliance among the local population.

According to some studies, the PR and PPV of FOBT for colorectal neoplasia in a screened population ranged from $4.8 \%$ to $12.9 \%$ and $13.3 \%$ to $42.7 \%$, respectively, ${ }^{(11,17-21)}$ which correlate well with our findings (PR 8.1\%; PPV 34.0\%). Importantly, more than half of the participants who had only one positive test in our study were subsequently diagnosed with colorectal neoplasia. This reinforces the importance of repeated screening with multiple stool samples to minimise the risk of false negative testing with FOBTs. Hyperplastic polyps were previously considered to be lesions with no malignant potential. However, recent studies suggest that hyperplastic polyps may represent precursor lesions of some sporadic colorectal cancers. ${ }^{(22)}$ Therefore, hyperplastic polyps were also included as colorectal neoplasia, together with adenomas and cancers, in our study. 
There are several limitations to this study. First, there was no follow-up data available for the participants who tested negative on FIT to ascertain whether these had been false negative results. This is a limitation in many similar studies on population screening programmes. Second, the compliance rate among participants was relatively low and nearly a quarter of all participants who tested positive on FIT refused further investigations. However, the authors contend that individuals who default on treatment are likely to be inherent in any screening programme.

In conclusion, the results of this pilot study hint at the potential obstacles that may surface during the implementation of future population-based screening programmes in Singapore. More extensive population education will be required to improve compliance with screening and tackle inhibitions that may be pervasive among the masses. Efforts may also be warranted to address feasibility concerns such as cost effectiveness.

\section{REFERENCES}

1. Seow A, Koh WP, Chia KS, et al. Trends in cancer incidence in Singapore, 1968-2002. Report No. 6. Singapore: Singapore Cancer Registry, 2004

2. Mandel JS, Bond JH, Church TR, et al. Reducing mortality from colorectal cancer by screening for fecal occult blood. Minnesota Colon Cancer Control Study. N Engl J Med 1993; 328:1365-71.

3. Hardcastle JD, Chamberlain JO, Robinson $\mathrm{MH}$, et al. Randomised controlled trial of faecal-occult-blood screening for colorectal cancer. Lancet 1996; 348:1472-7.

4. Kronborg O, Fenger C, Olsen J, Jorgensen OD, Sondergaard O. Randomised study of screening for colorectal cancer with faecal-occultblood test. Lancet 1996; 348:1467-71.

5. Jorgensen OD, Kronborg $\mathrm{O}$, Fenger $\mathrm{C}$. A randomised study of screening for colorectal cancer using faecal occult blood testing: results after 13 years and seven biennial screening rounds. Gut 2002; 50:29-32.

6. Lindholm E, Brevinge $\mathrm{H}$, Haglind E. Survival benefit in a randomized clinical trial of faecal occult blood screening for colorectal cancer. Br J Surg 2008; 95:1029-36.

7. Hewitson P, Glasziou P, Irwig L, Towler B, Watson E. Screening for colorectal cancer using the faecal occult blood test, Hemoccult. Cochrane Database Syst Rev 2007; 1:CD001216.

8. Goodyear SJ, Leung E, Menon A, et al. The effects of population-based faecal occult blood test screening upon emergency colorectal cancer admissions in Coventry and north Warwickshire. Gut 2008; 57:218-22.

9. Levi Z, Rozen P, Hazazi R, et al. A quantitative immunochemical fecal occult blood test for colorectal neoplasia. Ann Intern Med 2007; 146:244-55.

10. Vilkin A, Rozen P, Levi Z, et al. Performance characteristics and evaluation of an automated-developed and quantitative, immunochemical, fecal occult blood screening test. Am J Gastroenterol 2005; 100:2519-25.

11. Fu WP, Kam MH, Ling WM, et al. Screening for colorectal cancer using a quantitative immunochemical faecal occult blood test: a feasibility study in an Asian population. Tech Coloproctol 2009; 13:225-30.

12. Allison JE, Sakoda LC, Levin TR, et al. Screening for colorectal neoplasms with new fecal occult blood tests: update on performance characteristics. J Natl Cancer Inst 2007; 99:1462-70.

13. Burch JA, Soares-Weiser K, St John DJ, et al. Diagnostic accuracy of faecal occult blood tests used in screening for colorectal cancer: a systematic review. J Med Screen 2007; 14:132-7.

14. Chew MH, Suzanah N, Ho KS, et al. Colorectal cancer mass screening event utilising quantitative faecal occult blood test. Singapore Med J 2009; 50:348-53.

15. Chapple A, Ziebland S, Hewitson P, McPherson A. What affects the uptake of screening for bowel cancer using a faecal occult blood test (FOBt): a qualitative study. Soc Sci Med 2008; 66:2425-35.

16. Birkenfeld S, Belfer RG, Chared $M$, et al. Factors affecting compliance in faecal occult blood testing: a cluster randomized study of the faecal immunochemical test versus the guaiac faecal occult test. J Med Screen 2011; 18:135-41.

17. Ciatto S, Martinelli F, Castiglione G, et al. Association of FOBT-assessed faecal $\mathrm{Hb}$ content with colonic lesions detected in the Florence screening programme. Br J Cancer 2007; 96:218-21.

18. Castiglione G, Grazzini G, Ciatto S. Guaiac and immunochemical tests for faecal occult blood in colorectal cancer screening. Br J Cancer 1992; 65:942-4.

19. Castiglione G, Sala P, Ciatto $S$, et al. Comparative analysis of results of guaiac and immunochemical tests for faecal occult blood in colorectal cancer screening in two oncological institutions. Eur J Cancer Prev 1994; 3:399-405.

20. Denis B, Ruetsch M, Strentz P, et al. Short term outcomes of the first round of a pilot colorectal cancer screening programme with guaiac based faecal occult blood test. Gut 2007; 56:1579-84.

21. Guittet L, Bouvier V, Mariotte N, et al. Performance of immunochemical faecal occult blood test in colorectal cancer screening in average-risk population according to positivity threshold and number of samples. Int J Cancer 2009; 125:1127-33.

22. Do C, Bertrand C, Palasse J, et al. A new biomarker that predicts colonic neoplasia outcome in patients with hyperplastic colonic polyps. Cancer Prev Res (Phila) 2012; 5:675-84.

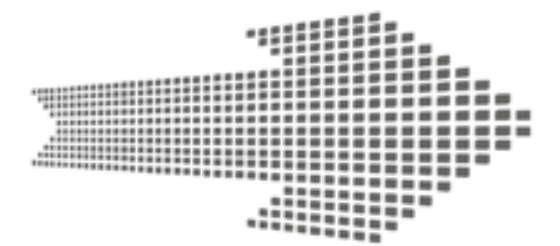

\section{Online Manuscript Submission www.editorialmanager.com/singaporemedj}

